\title{
Avaliação do impacto na infestação por Aedes aegypti em tanques de cimento do Município de Canindé, Ceará, Brasil, após a utilização do peixe Betta splendens como alternativa de controle biológico
}

\author{
Evaluation of the impact on Aedes aegypti infestation in cement tanks of the Municipal District \\ of Canindé, Ceará, Brazil after using the Betta splendens fish as \\ an alternative biological control
}

\author{
Luciano de Góes Cavalcanti Pamplona ${ }^{1,5}$, José Wellington de Oliveira Lima ${ }^{2,4}$, \\ Jane Cris de Lima Cunha ${ }^{3,4}$ e Eddie William de Pinho Santana ${ }^{1,4}$
}

\begin{abstract}
RESUM0
Canindé apresenta uma população de 71.235 habitantes. Em abril de 2001 iniciou a utilização de peixes larvófagos em tanques de cimento, localizados ao nível do solo, como forma de controle biológico para larvas de Aedes aegypti. Durante a visita do agente, ao invés de se tratar os tanques com larvicida, colocou-se um espécime do peixe Betta splendens por depósito. 0 presente trabalho teve como objetivo avaliar os resultados desta intervenção. Com os levantamentos do número de imóveis e depósitos existentes, estimou-se este número mês a mês determinando então o número de depósitos existentes por imóvel. Com esta estimativa e 0 número de imóveis visitados mensalmente analisou-se a infestação deste tipo de depósito. Em janeiro de 2001, 70,4\% dos tanques examinados apresentavam larvas; e apenas 7,4\% em janeiro de 2002. Em dezembro de 2002 este índice caiu para 0,2\%. Demonstrou-se com clareza a capacidade do Betta splendens como agente de controle biológico, em tanques de cimento, reduzindo 320 vezes a infestação deste tipo de recipiente de grande volume.
\end{abstract}

Palavras-chaves: Dengue. Aedes aegypti. Controle biológico. Peixe larvófago. Betta splendens.

\begin{abstract}
Canindé has a population of 71,235 inhabitants. In April 2001, the city started using larvivorous fish in cement water tanks, as a means of biological control of Aedes aegypti larvae. During house-to-house visits by health agents, instead of treating the water tank with larvicide, a Betta splendens fish was introduced into each tank. The number of houses and the number of tanks was estimated by monthly surveys. Then, the number of tanks per house was determined. Taking into account this estimated number and the number of houses visited, the infestation level for each kind of deposit was analyzed. In January 2001, 70.4\% of the water tanks presented mosquitoes. Following the intervention, in January 2002 only $7.4 \%$ were positive and by December 2002 the rate had dropped to $0.2 \%$. The efficacy of Betta splendens as a biological control agent in cement water tanks was clearly demonstrated, by achieving a 320 times reduction in the infestation level.
\end{abstract}

Key-words: Dengue. Aedes aegypti. Biological control. Larvivorous fish. Betta splendens.

Hoje 0 dengue causa cerca de 50 a 100 milhões de casos anuais e, 250 a 500 mil casos de dengue hemorrágico por ano, no mundo. 0 problema aumenta, pois é sabido que cerca de $50 \%$ da população mundial vivem em áreas endêmicas para 0 dengue ${ }^{23}$. A primeira epidemia de dengue no Ceará teve início em agosto de 1986, se estendendo até novembro de 1987. Neste período, foram notificados 26.938 casos com o pico de transmissão em abril de 1987, com 1674 casos de

\footnotetext{
1. Secretaria da Saúde do Ceará, Fortaleza CE. 2. Fundação Nacional de Saúde, Fortaleza CE. 3. Escola de Saúde Pública do Ceará, Fortaleza CE. 4. Universidade Estadual do Ceará, Fortaleza, CE. 5. Universidade Federal do Ceará, Fortaleza, CE.

Endereço para correspondência: Dr. Luciano Pamplona de Góes Cavalcanti. Rua Barão de Aratanha 999, José Bonifácio, $60050-070$ Fortaleza, CE.

Tel: 85252 4004, 99878969.

e-mail: lucianoc@saude.ce.gov.br

Recebido para publicação 6/1/2004

Aceito em 12/7/2004
} 
dengue clássico distribuídos em 52 municípios ${ }^{21}$. Os primeiros casos de dengue hemorrágico foram notificados no ano de 1994, quando foi detectada a introdução do sorotipo DEN-2. Neste ano, foram confirmados 25 casos com uma letalidade de $48 \%$, tendo sido considerada uma das mais elevadas do Brasil. Houve uma pausa na notificação de casos hemorrágicos e a partir de 1998, nenhum ano se passou sem que houvesse notificação; destacando-se 0 ano de 2000 com uma letalidade de $75 \%$.

Os primeiros registros do Aedes aegypti neste estado sugerem sua presença no ano de 1851, devido a uma grande epidemia de febre amarela onde foram relatados mais de 28.000 casos desta patologia atingindo mais da metade da população da época ${ }^{6}$. Em 1932 foram realizados no Estado, pela Fundação Rockefeller, os primeiros levantamentos de índices em alguns municípios. № período de 1931 a 1949 o Aedes aegypti foi encontrado em todos os municípios do estado $0^{13}$. Os focos do Aedes foram totalmente erradicados do estado no ano de 1950, e até 1983 a literatura dos órgãos oficiais não relata nenhum foco do mosquito. No ano de 1984, foi detectada a reintrodução do primeiro foco no estado, no Município de Aquiráz e desde este período não foi mais possível erradicá-lo do território Cearense $^{921}$. No ano de 1986, foi realizado um levantamento de índice de infestação onde identificou-se 65 municípios infestados pelo vetor. Em 1994, já haviam 112 municípios infestados e em 20010 estado já apresentava 169 (91,8\%) municípios com a presença do Aedes aegypti ( Dados fornecidos pela SESA).

0 Município de Canindé localiza se no Centro-Norte do Estado do Ceará, 4ํ 21' 32" (S) e 39ํ1' 42" (W), com 3.205,4 quilômetros quadrados, ficando a 127 quilômetros da capital Fortaleza. Apresenta limite com 15 municípios, todos infestados pelo Aedes aegypti. Possui uma população de 71.235 habitantes, com a maior parte na área urbana, e é uma cidade turística; sendo conhecida internacionalmente como 0 segundo maior santuário franciscano do mundo, chegando a receber anualmente cerca de 1 milhão de romeiros de todo 0 Brasil e de outros países (Secretaria Municipal de Turismo, 2002). Canindé apresenta um histórico recente de casos de dengue com 234 casos notificados em 1999; 175 em 2000 e, 467 em 2001. Em 2002, ocorreu uma redução bastante acentuada, tendo sido notificados apenas 82 casos de dengue. Com relação aos índices de infestação, estes se mantiveram baixos até 0 ano de 1998. A partir de então, os índices vêm se mantendo elevados. Pelo que foi descrito, Canindé atualmente é considerada como uma das mais importantes áreas de transmissão de dengue no Ceará, além do fato de ter sido neste município que em 2002 foi detectado pela primeira vez a circulação do sorotipo DEN-3 no Ceará.

Grande parte da dificuldade de controle deste vetor se deve a sua extraordinária competência na busca e escolha de locais preferenciais para oviposição. 0 Aedes aegypti mostra capacidade de colonizar os mais variados tipos de criadouros e, certamente, existe correlação entre os depósitos preferenciais para sua oviposição e os hábitos de armazenagem de água de cada população $0^{716}$. A atividade humana, seus hábitos, costumes e tradições somadas a outros fatores, tais como; topografia regional, temperatura, umidade e altitude exercem marcada influência nessa distribuição do vetor?.

Experimentos realizados em 1957 mostraram que os depósitos artificiais preferidos para postura são aqueles que apresentam superfície de água livre, paredes porosas e baixa reflectividade ${ }^{16}$. Alguns trabalhos na literatura citam que os pequenos depósitos artificiais são importantes para infestação por Aedes aegypti; entretanto outros autores mostram que em alguns locais os grandes reservatórios de água podem ser os mais infestados 345121415 . No nordeste do Brasil grande parte dos depósitos infestados são do tipo B; ou seja, grandes depósitos utilizados devido insuficiência no suprimento de água ${ }^{13}$. Dentre eles destacando-se tambores, tinas, toneis, potes e tanques ${ }^{13}$. No Ceará 0 Aedes aegypti mostrou preferência acentuada por depósitos com grande volume de água ${ }^{1}$. Em relação à qualidade da água mostrou-se preferência por meios pouco poluídos; contudo desde que as condições gerais fossem viáveis, tal seletividade se tornava duvidosa ${ }^{716}$. Vários trabalhos na literatura mostram que os depósitos predominantes variam de região para região e, principalmente de acordo com os hábitos da população, condições ambientais e com o fornecimento de água ${ }^{12811} 1819$. A tendência atual é restringir 0 uso de agentes químicos em depósitos de água que não podem ser eliminados ou manejados. Hoje portanto, a perspectiva de uma longa convivência com 0 Aedes aegypti nos leva a racionalização do emprego de inseticidas ${ }^{17}$. Neste sentido, o controle biológico e 0 manejo ambiental têm sido bastante incentivados.

Dentre os agentes de controle biológico em utilização em todo 0 mundo, os bacilos entomopatogênicos apresentam especial importância. No que se refere a utilização de espécies larvófagas no controle de insetos vetores de doenças, tais como malária, febre amarela e dengue, os peixes vêm sendo utilizados no mundo há muito tempo, principalmente nos criadouros naturais destes insetos ${ }^{22}$.

Foi realizado um experimento nas cidades de Tiputa e Avarotu, no atol Francês da Polinésia onde o Poecilia reticulata, espécie de peixe bastante difundida no mundo, foi indicado para ser utilizado em poços abertos, buracos com água e lagoas de acordo com a realidade local ${ }^{9}$. Esta utilização foi satisfatória em grande parte dos reservatórios, onde ele se adaptou rapidamente e proliferava sistematicamente eliminando as larvas ${ }^{9}$. Uma outra espécie exótica com grande capacidade de colonizar coleções d'água, mesmo aquelas com pouco teor de oxigênio e temperaturas elevadas, é 0 Betta ${ }^{20}$. É um peixe da família dos Anabantídeos, e apresenta grande tolerância a temperaturas da água em torno de $36^{\circ} C^{20}$.

A partir do final do ano 2000, a Secretaria de Estado da Saúde incorporou em caráter experimental o controle biológico com o peixe Betta no município de Fortaleza. № início de 2001, grande parte dos depósitos infestados no município de Canindé eram tanques de cimento (depósitos ao nível do chão; localizados no intra ou peridomicílio; feitos de cimento e freqüentemente com capacidade de armazenamento superior a 300 litros de água).

No mês de abril de 2001, iniciou-se a utilização do peixe Betta splendens nestes tanques de cimento como alternativa 
de controle biológico para formas imaturas do Aedes aegypti. Durante a visita de rotina do agente de endemias, ao invés de se tratar os tanques com o larvicida temefós colocou-se um espécime do peixe Betta em cada tanque com até 5.000 litros de água. Desta forma, o objetivo deste trabalho foi avaliar os resultados de uma intervenção com o peixe Betta splendens para controlar formas imaturas do Aedes aegypti, em tanques de cimento do município de Canindé, Ceará.

\section{MATERIAL E MÉTODOS}

No período de janeiro de 2001 a dezembro de 2002, a equipe de controle de endemias do município de Canindé realizou dez ciclos de visitas domiciliares para tratamento e inspeção dos imóveis, sendo quatro em 2001 e seis em 2002, totalizando 69.596 visitas domiciliares. Nestes dez ciclos foram realizadas 9.213 inspeções, e 2.702 imóveis estavam infestados pelo Aedes aegypti, no momento da visita. A partir de abril de 2001, cada agente de endemias quando encontrava um tanque de cimento com capacidade superior a 200 litros de água colocava um espécimen do peixe Betta por depósito. A substituição do larvicida por este peixe foi uma alternativa aceita pelo responsável do imóvel no momento da visita. Caso o peixe não fosse encontrado na próxima visita era reposto pelo agente.

Foram trabalhados os 3 bairros mais (23,7\%) populosos e que apresentavam maior $(33,1 \%)$ número de imóveis que foram Can, Matadouro e Alto do Custódio. Analisou-se cerca de 2.000 formulários (etiqueta FAD-02) utilizados na rotina do Programa de Controle do Dengue, para o envio de espécimes ao laboratório.

0 município de Canindé realiza anualmente, no mês de março, um levantamento para determinar o número de imóveis e depósitos existentes, conforme a Tabela 1. Calculou-se o número estimado mensal de imóveis e depósitos entre janeiro de 2001 e dezembro de 2002, conforme a Tabela 2. Ciente desta estimativa do número de depósitos existentes por imóvel e o número de imóveis visitados, coletados das fichas preenchidas pelos agentes de saúde a cada mês; além do número e do tipo de depósitos infestados foi possivel descrever a infestação para cada tipo de depósito. Optou-se, ainda, por trabalhar os dados dos meses de janeiro, abril, julho, outubro e dezembro por apresentarem aproximadamente 0 mesmo número de imóveis visitados nos dois anos, 0 que facilitou a análise e a interpretação dos dados entre os mesmos períodos.

\section{RESULTADOS}

Até 0 final do período analisado haviam sido peixados 2.071 tanques, correspondendo a 79,5\% do total de tanques na área estudada que não puderam ser vedados ou eliminados dos domicílios.

A Tabela 3 mostra uma acentuada diminuição da infestação nos tanques de cimento após a substituição da utilização do larvicida químico temefós pela espécie de peixe Betta splendens. No mês de janeiro de 2001, 70,4\% dos
Tabela 1 - Levantamento do número de depósitos existentes nos bairros Can, Matadouro e Alto do Custódio, no período de 2001, 2002 e 2003.

\begin{tabular}{lccccc}
\hline $\begin{array}{l}\text { Depósito } \\
\text { (no existente) }\end{array}$ & 2001 & 2002 & 2003 & $\begin{array}{c}\text { Variação mensal } \\
2001 / 2002\end{array}$ & $\begin{array}{c}\text { Variação mensal } \\
2002 / 2003\end{array}$ \\
\hline Imóveis & 5.695 & 5.806 & 5.988 & 0,001609 & 0,002572 \\
Tanques & 2.477 & 2.659 & 2.824 & 0,005908 & 0,005017 \\
Caixas d'água & 2.437 & 2.722 & 2.932 & 0,009217 & 0,006193 \\
Potes ou filtros & 6.958 & 7.579 & 8.015 & 0,007124 & 0,004661 \\
Outros & 1.874 & 1.771 & 1.765 & $-0,004711$ & 0,000283 \\
\hline
\end{tabular}

Tabela 2 - Estimativa do número de depósitos existentes, mês a mês, nos bairros Can, Matadouro e Alto do Custódio no período dejaneiro de 2001 a dezembro de 2002.

\begin{tabular}{lccccc}
\hline Mês/ano & № de imóveis & № de tanques & № de caixas & № de potes & № de outros \\
\hline Janeiro/2001 & 5.677 & 2.462 & 2.393 & 6.859 & 1.892 \\
Abril/2001 & 5.704 & 2.492 & 2.459 & 7.008 & 1.865 \\
Julho/2001 & 5.731 & 2.537 & 2.528 & 7.159 & 1.838 \\
Outubro/2001 & 5.758 & 2.582 & 2.599 & 7.313 & 1.811 \\
Dezembro/2001 & 5.776 & 2.612 & 2.647 & 7.417 & 1.794 \\
Janeiro/2002 & 5.785 & 2.627 & 2.671 & 7.470 & 1.786 \\
Abril/2002 & 5.821 & 2.672 & 2.739 & 7.614 & 1.770 \\
Julho/2002 & 5.866 & 2.712 & 2.790 & 7.721 & 1.767 \\
Outubro/2002 & 5.911 & 2.754 & 2.841 & 7.829 & 1.766 \\
Dezembro 2002 & 5.941 & 2.782 & 2.877 & 7.902 & 1.766 \\
\hline
\end{tabular}

Tabela 3 - Estimativa do número de tanques por imóvel e \% de tanques infestados por Aedes aegypti, no período entre janeiro de 2001 e dezembro de 2002.

\begin{tabular}{lccccc}
\hline Mês/ano & $\begin{array}{c}\text { Imóveis } \\
\text { vistoriados }\end{array}$ & $\begin{array}{c}\text { № de tanques } \\
\text { por imóvel }\end{array}$ & $\begin{array}{c}\text { № de tanques } \\
\text { vistoriados }\end{array}$ & $\begin{array}{c}\text { № de tanques } \\
\text { infestados }\end{array}$ & $\begin{array}{c}\text { Tanques } \\
\text { infestados (\%) }\end{array}$ \\
\hline Janeiro/2001 & 910 & 0,4337 & 395 & 278 & 70,4 \\
Abril/2001 & 934 & 0,4369 & 408 & 183 & 44,8 \\
Julho/2001 & 903 & 0,4427 & 400 & 156 & 39,0 \\
Outubro/2001 & 989 & 0,4484 & 443 & 36 & 8,1 \\
Dezembro/2001 & 905 & 0,4522 & 409 & 10 & 2,4 \\
Janeiro/2002 & 920 & 0,4541 & 418 & 31 & 7,4 \\
Abril/2002 & 930 & 0,4590 & 427 & 13 & 3,0 \\
Julho/2002 & 898 & 0,4623 & 415 & 8 & 1,9 \\
Outubro/2002 & 944 & 0,4659 & 440 & 10 & 2,3 \\
Dezembro/2002 & 950 & 0,4683 & 445 & 1 & 0,2 \\
\hline
\end{tabular}

tanques examinados apresentavam-se com larvas do mosquito Aedes aegypti, enquanto janeiro de 2002, apenas 7,4\% destes tanques apresentava a presença do vetor. Esta diminuição da infestação manteve-se bastante acentuada quando comparados os mesmos períodos nos dois anos consecutivos, de acordo com a Tabela 3. Esta mesma Tabela foi elaborada para caixas d'água, potes e outros depósitos revelando as seguintes infestações conforme a Tabela 4.

Todos os reservatórios de água apresentaram diminuição da infestação, entretanto bem menos acentuada do que nos tanques. Em relação aos potes de barros e/ou filtros, que são os depósitos mais freqüentemente encontrados, aproximadamente, em uma proporção de 1,3 depósitos/ imóvel, a redução foi de 6,3\% para 0,4\% infestados revelando uma diminuição mais discreta da infestação. 
Tabela 4 - Estimativa da \% de caixas, potes e outros depósitos infestados por Aedes aegypti, no período entre janeiro de 2001 e dezembro de 2002.

\begin{tabular}{lccc}
\hline Mês/ano & Caixas infestadas (\%) & Potes infestados (\%) & Outros infestados (\%) \\
\hline Janeiro/2001 & 30,2 & 6,3 & 23,8 \\
Abril/2001 & 19,3 & 5,5 & 19,7 \\
Julho/2001 & 17,1 & 6,6 & 10,0 \\
Outubro/2001 & 7,4 & 4,1 & 3,5 \\
Dezembro/2001 & 1,7 & 1,3 & 1,8 \\
Janeiro/2002 & 5,9 & 4,8 & 7,0 \\
Abril/2002 & 3,6 & 2,1 & 1,4 \\
Julho/2002 & 2,1 & 2,4 & 2,6 \\
Outubro/2002 & 2,4 & 3,0 & 2,8 \\
Dezembro/2002 & 0,6 & 0,4 & 0,7 \\
\hline
\end{tabular}

A Figura 1 mostra a redução da infestação por Aedes aegypti em cada tipo de depósito entre o período de janeiro de 2001 e dezembro de 2002.

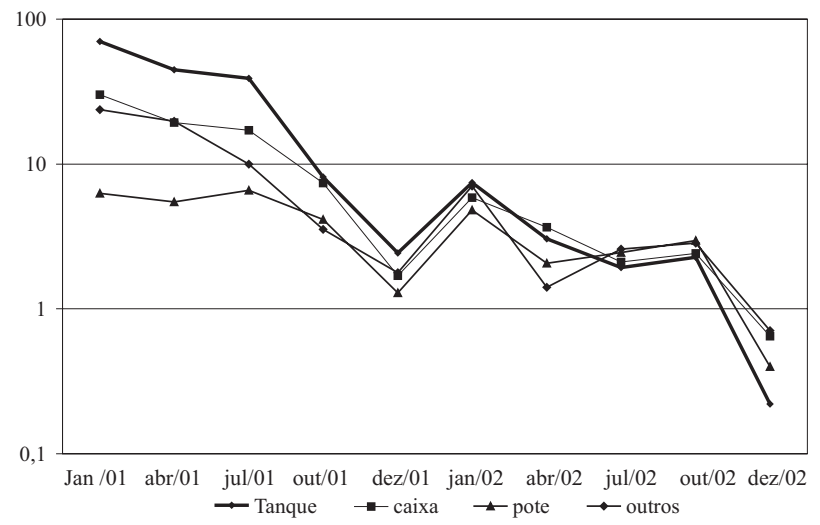

Figura 1 - Infestação por Aedes aegypti em tanques de cimento, caixas d'água, potes e outros depósitos, no município de Canindé, entre janeiro de 2001 a dezembro de 2002.

Em relação aos tanques, onde foram introduzidos os peixes, demonstrou-se claramente a redução significativa da infestação ( 320 vezes) enquanto a redução da infestação nas caixas ( 46 vezes), e nos potes ( 16 vezes) foi bem mais sensível. Já nos outros tipos de depósitos a infestação diminuiu em torno de 33 vezes, entretanto a respeito deste tipo de reservatório (outros) é importante deixar claro que eles não apresentam uma contagem tão fidedigna, devido principalmente a dificuldade em identificá-los na sua totalidade, já que os mesmos só são contabilizados se estiverem com água.

\section{DISCUSSÃ0}

Demonstrou-se com clareza a capacidade do Betta splendens como agente de controle biológico para formas imaturas de Aedes aegypti, em tanques de alvenaria. Observou-se ainda que a redução da infestação ocorrida nos outros tipos de depósitos deve-se, provavelmente, ao fato da diminuição da população de mosquitos, devido a predação das larvas pelo peixe, e não apenas a mudança de local de postura como ocorre quando se veda um depósito ou se elimina 0 mesmo do ambiente doméstico. Desta forma, a utilização desta espécie de peixe se mostrou mais interessante do que a utilização dos larvicidas convencionais, pois foi capaz de baixar as infestações sem a necessidade de utilização de componentes químicos no ambiente. Seria ainda importante mesmo com resultados similares a utilização de inseticidas químicos, já que na atualidade busca-se cada vez mais diminuir o uso destes químicos.

Além disso, para que esta tecnologia de controle apresente resultados sustentáveis é extremamente importante que as comunidades se apropriem deste método, não só acreditando na sua eficácia mas contribuindo de forma direta; principalmente repondo os peixes que por algum motivo tenham morrido ou tenham sido retirados dos tanques. Através de trabalhos de mobilização social e educação em saúde é possível informar a população das vantagens desta estratégia e principalmente consultá-la acerca da adoção desta ou de outra medida de controle. Com as ações de controle de endemias descentralizadas para os municípios é importante coordená-las de forma direcionada para cada localidade, devendo atuar quase que de forma personalizada em cada situação. Só assim será possível uma reduçã̃o sustentável da infestação.

\section{AGRADECIMENTOS}

A Dra. Rosélia Maria Mesquita, ao Sr. José Maria Ferreira e a todos os técnicos da 5 a CERES por seu incentivo e colaboração no desenvolvimento deste trabalho. A todos os técnicos, supervisores e agentes de endemias da secretaria municipal de saúde de Canindé pelos relatos das ações e colaboração no trabalho.

\section{REFERÊNCIAS BIBLIOGRÁFICAS}

1. Bezerra HSS. Determinantes da infestação domiciliar pelo Aedes aegypti na cidade de Fortaleza. Dissertação de Mestrado, Universidade Federal do Ceará, Fortaleza, CE, 1999.

2. Bond HÁ, Fay RW. Factors influencing Aedes aegypti occurrence in containers. Mosquito News 30: 394-402, 1969.

3. Chadee DD. Aedes aegypti surveillance in Tobago, West Indies [(1983-198) ]. Journal American Mosquito Control Association 6: 148-150, 1990.

4. Cruz AM, Mesa A, Martín JLS. La comunidad y el control de Aedes aegypti: percepción y comportamiento respecto al larvicida abate. Revista Cubana de Medicina Tropical 53: 44-47, 2001.

5. Focks DA, Chadee DD. Pupal survey: an epidemiologically significant surveillance method por Aedes aegypti: an example using data from Trinidad. American Journal Tropical Medicine and Higyene 56: 159-167, 1997.

6. Franco 0. História da febre amarela no Brasil. Rio de Janeiro, RJ, 1976.

7. Gadelha DP, Toda AT. Biologia e comportamento do Aedes aegypti. Revista Brasileira de Malariologia de Doença Tropical 37: 29-36, 1985.

8. Kittayapong P, Strickman D. Distribution of container-inhabiting Aedes aegypti larvae (Diptera: Culicidae) at a dengue focus in Thailand. Journal Medical Entomology 30: 601-606, 1993.

9. Lardeux F, Sechan Y, Loncke S. Integrated control of peridomestic larval habitats of Aedes and Culex mosquitoes ( Diptera: Culicidae) in atoll villages of French Polynesia. Journal Medical Entomology 39: 493-498, 2002.

10. Lima MM, Aragão MB, Amaral RS. Criadouros de Aedes aegypti encontrados em alguns bairros da cidade do Rio de Janeiro, RJ, Brasil, em 1984 1985. Cadernos de Saúde púbilca 4: 293-300, 1985. 
11. Marcoris MLG, Mazine CAB, Andrighetti MTM. Factors favoring houseplant container infestation with Aedes aegypti larvae in Marília, São Paulo, Brazil. Pan American Journal Public Health 1(4), 1997.

12. Marquetti MC, Carús F, Aguilera L, Gonzalez D, Navarro A. Comportamiento del programa de erradicación de Aedes aegypti en 2 municipios de Ciudad de la Habana, 1990-1992. Revista Cubana de Medicina Tropical 48: 174-177, 1996.

13. Ministério da Saúde. Programa Nacional de Controle da Dengue (PNCD), Brasília, DF, 2002.

14. Nathan MB, Giglioli ME. Erradicación de Aedes aegypti en Caimán Brag y pequenõ Caimán, Antilhas Britânicas, con abate ( temefos) en 1970-1972. Boletin of Sanitaria Panamericana 92: 18-32, 1973.

15. Nathan MB, Knudsen AB. Aedes aegypti infestation characteristics in several Caribbean countries and implications for integrated community based control. Journal American Mosquito Control Association 7: 400-404, 1991.

16. O'gower AK. The influence of the surface on oviposition by Aedes aegypti (Linn.) (Diptera: Culicidae). Proceedings of the Linnean Society of New South Wales 82: 240-244, 1957.

17. Pan-American Health Organization. Dengue and dengue hemorrhagic fever in the Americas: guidelines for prevention and control. ( Scientific Publication, n. 548), Washington, 1994.
18. Pereira M. Recipientes artificiais utilizados como criadouros por Aedes aegypti na região de Araçatuba, São Paulo. Dissertação de Mestrado, Escola de Saúde Pública da Universidade de São Paulo, SP, 1996.

19. Pinheiro VCS, Tadei WP. Frequency, diversity and productivity study on the Aedes aegypti most preferred containers in the city of Manaus, Amazonas, Brazil. Revista do Instituto de Medicina Tropical de São Paulo 44: 245-250, 2002.

20. Santana EWP, Pamplona LGC, Oliveira-Lima JW, Ribeiro ZM, Regazzi ACF. Biological Control of Aedes aegypti I. Evaluation of the larvivorous potential of the Betta splendens in water boxes in Fortaleza, Ceará, Brasil. In: Resumos do XXXVI Congresso da Sociedade Brasileira de Medicina Tropical p. 37, 2001.

21. Secretaria de Saúde do Estado do Ceará. Boletim epidemiológico. Disponível na Internet através do site: www.saude.ce.gov.br, 2002.

22. World Health Organization. Division of Control of Tropical Diseases. [ on line]. Dengue and dengue hemorrhagic fever. Disponível na internet via www.who.int/inf-fs/en/fact17html, 1986.

23. World Health Organization. Dengue and dengue hemorrhagic fever. Fact Sheet No 117, revised April 2002. Disponível na internet via www.who.int/ csr/disease/en/who, 2002. 